\title{
Die Verteilung des Bodens mitbestimmen
}

Rezension zu Brigitta Gerber / Ulrich Kriese (Hg.) (2019): Boden behalten - Stadt gestalten. Zürich: rüffler \& rub.

Isaak Granzer

Abb. 1 Titel des

Buches (Quelle: Verlag rüffler \& rub)

BODEN BEHALTEN STADT OESTALTEN
Die Vergesellschaftung von Grund und die Eigentumsfrage in der Wohnungspolitik ist eine seit Jahren wiederkehrende und gleichzeitig hochaktuelle Debatte. Diskussionen wie die um den Berliner Mietendeckel und Initiativen wie „Deutsche Wohnen \& Co. enteignen“ zeigen nicht nur die politische und juristische Komplexität des Themas, sondern auch die Emotionalität der Debatte und seine sozialen wie gesellschaftlichen Aspekte (vgl. Der Spiegel 2021). Mit Boden behalten - Stadt gestalten (2019a) liegt ein inspirierender Sammelband der Herausgebenden Brigitta Gerber und Ulrich Kriese vor, der aus der Praxis berichtet und fragt: Wo steht heute „die Bodenfrage“? Wem soll Grund und Boden gehören? Wie sehen Alternativen zum renditeorientierten Wohnungsbau aus? Wie kann nicht-profitorientierter Wohnbau erfolgen und welche Rechtsinstrumente bieten sich dafür an? Wie können diese auf die politische Agenda gebracht und eingefordert werden? Und wie profitieren Städte und Kommunen langfristig davon?

Als Antwort auf diese Fragen plädieren die versammelten Texte nicht ausschließlich, aber vor allem für das (Erb-)Baurecht[1]: Dieses erlaubt die Bebauung eines Grundstücks gegen Zins, ohne das Grundeigentum abgeben zu müssen. Beispielsweise erhalten Genossenschaften auf diese Weise bezahlbares Bauland von einer Kommune, ohne dass kommunales Eigentum aus der Hand gegeben wird. Im Sammelband werden unter anderem folgende Fragen behandelt: Was kann (Erb-)Baurecht leisten? Welche Vorteile ergeben sich daraus für die (Zivil-)Gesellschaft? Und: Wie kann es eingesetzt werden? Insbesondere letzterer Aspekt macht den großen Reiz vieler Beiträge aus. Ergänzt werden sie durch Ausführungen zum städtischen Bodenmanagement und gemeinnützigen Wohnungsbau. Gerber und Kriese vereinen dazu in diesem Band verschiedene Perspektiven von mehr als 35 Autor_innen.

Das Buch ist in acht Abschnitte gegliedert. Einer kurzen Einführung folgend, wird im Abschnitt „Bodenpolitische Herausforderungen“ der Kontext aktueller und vergangener Stadtentwicklungspolitik skizziert. Auch globale Dimensionen der Problematik werden hier angerissen, etwa internationale Verflechtungen von Landkäufen oder die problematische Funktion des Bodens als Investitionsobjekt und Kapitalanlage. 
In den Abschnitten „Gemeinwohlorientierter Umgang mit Liegenschaften und mit Grund und Boden: Modelle und Erfahrungen“ sowie „Geschichte des Bodeneigentums und des Bodenrechts“ wird eine historische Perspektive auf Eigentum und den Umgang mit Boden gegeben. Aktuelle gemeinwohlorientierte Organisationsformen werden überblicksartig dargestellt. Die Beiträge machen deutlich, dass die „Bodenfrage“ und damit die Frage nach kommunalem bzw. staatlichem Grundeigentum keine neue ist, sondern Stadtpolitiken in vielen gesellschaftlichen Kontexten seit Jahrzehnten begleitet. Besonders hervorzuheben ist hier Andrew Purves' Beitrag, der die Situation in Hongkong und Singapur beleuchtet, die sich deutlich von derjenigen in europäischen Staaten unterscheidet: In diesen Stadtstaaten wurde ein Mittelweg zwischen öffentlicher und privater Landnutzung gewählt, wobei sich Grundeigentum (zu einem großen Teil) in staatlichem Besitz befindet (Purves 2019: 93 f.).

Im zweiten Teil der Publikation rückt das Instrument des (Erb-)Baurechts selbst in den Fokus: Im Abschnitt „Das (Erb-)Baurecht: Ein zentrales bodenpolitisches Instrument" werden dieses Rechtsinstrument und seine Anwendungsmöglichkeiten anhand konkreter Beispiele näher erläutert. Deutlich wird, dass durch das (Erb-)Baurecht Projekte zur Umsetzung gelangen konnten, deren Realisierung in einer renditeorientierten Marktlogik nicht denkbar wäre. Die Kommunen erhalten durch die Vergabe im (Erb-)Baurecht Steuerungsmöglichkeiten, die bei einem Verkauf nicht oder nur schwer zu garantieren sind. Da sie weiterhin Grundeigentümerinnen sind, bleibt ihnen - etwa durch Konzeptvergaben - somit ein gewisser Gestaltungsspielraum innerhalb der Stadtentwicklung, etwa auch die Möglichkeit der Förderung gesellschaftspolitischer Anliegen.

Ein besonderer Fokus des Sammelbands liegt auf der wohnungspolitischen Situation in Basel, wo es eine lange Tradition des Einsatzes bodenpolitischer Maßnahmen gibt. Im Abschnitt „Bodenpolitische Erfahrungen, Vorbilder \& Akteure in Basel“ werden Projekte, die das Instrument des Baurechts anwenden, wie Bodenstiftungen oder das dortige Mietshäuser Syndikat und weitere Instrumente wie die Basler Mehrwertabgabe, aus verschiedenen Blickwinkeln beleuchtet. Anschließend werden die Basler Bodeninitiative, ihr nachfolgende Schweizer Initiativen sowie verschiedene bodenpolitische Bewegungen in Deutschland vorgestellt.

Dadurch, dass Gerber und Kriese wohnungs- und bodenpolitische Aspekte des Rechtsinstruments (Erb-)Baurecht aus verschiedenen Blickwinkeln beleuchten, wird dessen großes Potenzial deutlich. Die verschiedenen Herangehensweisen an das Thema - seien sie historisch, ökonomisch oder politisch-aktivistisch - werden sehr überzeugend zu einem umfassenden Plädoyer für das (Erb-)Baurecht vereint.

Gleichzeitig stößt der Sammelband hier an seine Grenzen: Er ist weder ein Lehrbuch, das eine umfassende Analyse zu Grundeigentum und (Erb-) Baurecht bietet, noch eine Aufsatzsammlung, die sich der Analyse der politischen und sozialen Dimension der präsentierten Initiativen verschreibt. Zielgruppe des Buchs sind explizit Personen, die sich bodenpolitisch engagieren möchten - der Band soll als „Handreichung und Materialsammlung"(Gerber/Kriese 2019b: 11) dienen und versteht sich nicht als wissenschaftliche Aufsatzsammlung. So wird aus Verträgen zitiert, es 
werden konkrete Forderungstexte vorgestellt und Gründe dargelegt, warum manche der präsentierten Initiativen schlussendlich an der Urne, also an der Abstimmung der Bevölkerung, scheiterten.

Der Band bietet dennoch wertvolles Material für die wissenschaftliche Einordnung und Behandlung lokaler bodenpolitischer Initiativen sowie des Diskurses über Wohnungs- und Bodenpolitik. Durch die Breite der behandelten Themen kann es als vielfältige Quelle zu politischen Initiativen bzw. aktivistischen Projekten dienen, auch weil Initiativen, die bisher nur lokal große Beachtung fanden, präsentiert werden (wie beispielsweise die Zürcher PWG-Stiftung, vorgestellt von Kornel Ringli).

Die oftmals mit Verweis auf institutionelle und (verfassungs-)rechtliche Einschränkungen als alternativlos dargestellte, in einer neoliberalen Marktlogik agierende Wohnungsversorgung hat in den vergangenen Jahren zu vermehrtem Aktivismus und wohnungspolitischen Konflikten geführt, die in der Stadtforschung bereits zunehmend Aufmerksamkeit erhalten. Hier bringt der Band allerdings nur die Sicht der Praxis ein: Die Erfahrungen der verschiedenen Initiativen sind gleichzeitig Dokumentation und Inspirationsquelle für neue Bewegungen, allerdings keine wissenschaftlichen Analysen, wie sie sich beispielsweise bei Anne Vogelpohl, Lisa Vollmer, Elodie Vittu und Norma Brecht (Vogelpohl et al. 2017) oder Lisa Vollmer (2019) finden.

Diese praktische Sicht schließt jedoch Anknüpfungspunkte zur bisherigen Forschung nicht aus. Das Buch kann als Kritik an der zunehmenden unternehmerischen Orientierung von Stadtpolitiken und deren neoliberalen Ausrichtung zur „unternehmerischen Stadt“ verstanden werden (vgl. dazu die Überlegungen von Harvey 1989; Häußermann/Läpple/Siebel 2008). „Investitionsfreundliche Politiken“ führten - wie etwa von Andrej Holm(2011) dargelegt - zu einem systematisch bedingten Mangel an leistbaren Wohnungen und damit einhergehend zu sozialer und räumlicher Polarisierung. Der Sammelband möchte - anknüpfend an Positionspapiere wie die Bodenpolitische Agenda 202O-203O des Deutschen Instituts für Urbanistik und des Bundesverbands für Wohnen und Stadtentwicklung (2017) oder die Empfehlungen der Österreichischen Raumordnungskonferenz (2017) weniger eine Analyse dieser Probleme bieten, sondern vielmehr den Blick auf Lösungsmöglichkeiten richten, eben in Form einer bereits existierenden Wohnungspolitik des Gemeinwesens.

Die Förder_innen des (Erb-)Baurechts bieten eine Gegenperspektive zur spätkapitalistischen unternehmerischen Stadt: genossenschaftlich organisierte Institutionen, die rein monetäre oder gar spekulative Politiken zurückdrängen und Wohnungen so dem Markt entziehen. Dennoch stellen die präsentierten Initiativen und Organisationsformen im heutigen Wohnungswesen weiterhin ein Randphänomen dar, obwohl sie einen sehr pragmatischen Weg einschlagen: Sie zeigen Möglichkeiten auf, wie in den bestehenden rechtlichen, administrativen und marktwirtschaftlichen Strukturen agiert werden kann. Es darf nicht vergessen werden, dass das (Erb-) Baurecht nicht ohne weiteres gesellschaftliches oder politisches Zutun alle gesellschaftlichen Schichten inkludiert. Zentral ist die Überlegung, Boden als Gemeingut wahrzunehmen - und dieses politisch als auch gesellschaftlich so zu behandeln (Gerber/Kriese 2019c: 417). Wiederholt wird klargestellt, 
dass die Wohnungsfrage eigentlich eine Bodenfrage ist, und es wird aus verschiedenen Perspektiven dargelegt, dass ,[d]ie Privatisierung von Boden [...] eine der Grundursachen sozialer Ungerechtigkeit“ ist (Benthaus/Heimann 2019: 32).

Bei der Lektüre drängen sich verschiedenste Anknüpfungspunkte auf, die, losgelöst vom konkreten Rechtsinstrument (Erb-)Baurecht, die Möglichkeit gesellschaftlicher Veränderungen konkret erscheinen lassen: Können die hier um den Rahmen des (Erb-)Baurechts herum gezeigten Organisationsformen erste Schritte hin zu einer gesellschaftlichen Produktion des Urbanen sein? Wie können die präsentierten Vorhaben inklusiver gedacht und gemacht werden? Denn schlussendlich sollte eine Stadt für alle ohne ökonomische Hürden auskommen. Was lässt sich dabei aus den bisherigen zivilgesellschaftlichen Aktivitäten wie der Bodeninitiative lernen? Oder aus einer banalen, realpolitischen Sicht gefragt: Wieso ist das Konzept des (Erb-) Baurechts nicht schon längst ein allgemein verbindlicher Standard in Zeiten der Wohnungskrise?

Die Herausgebenden bezeichnen Boden behalten - Stadt gestalten als „ein Buch für alle, die über die Verteilung des Bodens mitbestimmen wollen“. Dem ist uneingeschränkt zuzustimmen. Die Aufsätze sind vielschichtig, durchgehend allgemein verständlich und prägnant verfasst. Das Buch ist darüber hinaus noch viel mehr: Für Forschende kann es als Inspirationsquelle für wohnungs- und bodenpolitische Fragestellungen dienen.

\section{Endnoten}

[1] In Deutschland wird dieses Recht als Erbbaurecht bezeichnet, in der Schweiz (und in Österreich) als Baurecht. Der Sammelband umfasst sowohl deutsche als auch Schweizer Perspektiven und verwendet die Schreibweise (Erb-)Baurecht, mit der versucht wird, beide Begriffe abzubilden. Die vorliegende Rezension übernimmt daher diese Schreibweise.

\section{Autor_innen}

Isaak Granzer studierte Raumplanung und Raumordnung sowie Philosophie in Wien. Er forschte zu den Themen internationale Stadtentwicklung sowie zum Einsatz von Sprache und Bild in planerischen Projekten.

isaak.granzer@icloud.com

\section{Literatur}

Benthaus, Sebastian / Heimann, Hans-Georg (2019): Betongold. Immobilienpolitik der Pensionskassen. In: Brigitta Gerber / Ulrich Kriese (Hg.), Boden behalten - Stadt gestalten. Zürich: rüffler \& rub, 31-41.

Der Spiegel (2021): Berliner Enteignungs-Initiative hat bereits 130.000 Unterschriften gesammelt. In: Der Spiegel, 26.4.2021. https://www.spiegel.de/wirtschaft/soziales/ deutsche-wohnen-und-co-enteignen-berliner-initiative-nimmt-kurs-auf-volksentscheida-07536610-88ca-41fa-af37-96c58e468306 (letzter Zugriff am 2.5.2021).

Deutsches Institut für Urbanistik / Bundesverband für Wohnen und Stadtentwicklung (Hg.) (2017): Bodenpolitische Agenda 2020-2030. Warum wir für eine nachhaltige und sozial gerechte Stadtentwicklungs- und Wohnungspolitik eine andere Bodenpolitik brauchen. Berlin. 
Gerber, Brigitta / Kriese, Ulrich (Hg.) (2019a): Boden behalten - Stadt gestalten. Zürich: rüffler \& rub.

Gerber, Brigitta / Kriese, Ulrich (2019b): Einführung. In: Brigitta Gerber / Ulrich Kriese (Hg.), Boden behalten - Stadt gestalten. Zürich: rüffler \& rub, 8-15.

Gerber, Brigitta / Kriese, Ulrich (Hg.) (2019c): Fazit und Empfehlungen. In: Brigitta Gerber / Ulrich Kriese (Hg.), Boden behalten - Stadt gestalten. Zürich: rüffler \& rub, 416-422.

Harvey, David (1989): From managerialism to entrepreneurialism: The transformation in urban governance in late capitalism. In: Geografiska Annaler. Series B, Human Geography 71/1, 3-17.

Häußermann, Hartmut / Läpple, Dieter / Siebel, Walter (2008): Stadtpolitik. Frankfurt am Main: Suhrkamp.

Holm, Andrej (2011): Wohnung als Ware. Zur Ökonomie und Politik der Wohnungsversorgung. In: Widersprüche: Zeitschrift für sozialistische Politik im Bildungs-, Gesundheitsund Sozialbereich 31/121, 9-20.

ÖsterreichischeRaumordnungskonferenz(Hg.)(2017): Flächensparen, Flächenmanagement \& aktive Bodenpolitik. Ausgangslage, Empfehlungen \& Beispiele. ÖROK-Empfehlung Nr. 56. Wien.

Purves, Andrew (2019): Die Modelle Hongkong und Singapur. Staatliche Landverpachtung statt Verkauf. In: Brigitta Gerber / Ulrich Kriese (Hg.), Boden behalten - Stadt gestalten. Zürich: rüffler \& rub, 92-104.

Vogelpohl, Anne / Vollmer, Lisa / Vittu, Elodie / Brecht, Norma (2017): Die Repolitisierung des Wohnens. Städtische soziale Bewegungen für ein Recht auf Wohnen und auf Stadt in Hamburg, Berlin, Jena und Leipzig. In: Barbara Schönig / Justin Kadi / Sebastian Schipper (Hg.), Wohnraum für alle?! Perspektiven auf Planung, Politik und Architektur. Bielefeld: transcript, 105-130.

Vollmer, Lisa (2019): Mieter_innenbewegungen in Berlin und New York. Die Formierung politischer Kollektivität. Wiesbaden: Springer VS. 